\title{
Análisis de flujo de ventilación mediante software de CFD como mecanismo de conservación del patrimonio, aplicado a la Antigua Capitanía de Puerto Limón
} Ventilation flow analysis using CFD software as a heritage conservation tool, applied to the "Antigua Capitanía" of Port Limon

\section{Enmanuel Salazar-Ceciliano ${ }^{1}$}

Salazar-Ceciliano, E. Análisis de flujo de ventilación mediante software de CFD como mecanismo de conservación del patrimonio, aplicado a la Antigua Capitanía de Puerto Limón. Tecnología en Marcha. Edición especial. Escuela de Arquitectura y Urbanismo, Diciembre 2020. Pág 61-70.

doi https://doi.org/10.18845/tm.v33i8.5509 


\title{
Palabras clave
}

Patrimonio cultural; arquitectura tradicional, conservación de los bienes culturales, humedad; viento; bioclimatología.

\section{Resumen}

Los hongos dificultan los procesos de conservación de bienes patrimoniales dado a que originan su biodeterioro. Las esporas producidas por estos organismos, las cuales también pueden inducir a complicaciones de salud en los usuarios del inmueble, dependen de la humedad acumulada en los materiales para poder germinar, por lo que controlar las condiciones ambientales resulta determinante si se desea potenciar al máximo cada intervención realizada. Aplicaciones de dinámica de fluidos asistida por computadora (CFD por sus siglas en inglés) que han sido tradicionalmente utilizados para simulaciones energéticas, están siendo empleados para determinar las corrientes de aire dentro de los espacios y con esto propiciar estrategias de conservación basadas en la ventilación natural.

Este artículo tiene como objetivo mostrar la metodología empleada para el análisis de flujos de aire dentro de la Antigua Capitanía del Puerto de Limón en Costa Rica, utilizando el software AUTODESK FlowDesign y la estrategia de análisis de datos desarrollada por Víctor Fuentes Freixanet y Manuel Rodríguez Viqueira. El documento tiene como marco el proyecto de investigación "Conservación de la arquitectura caribeña costarricense a partir de la aplicación de técnicas de avanzada para el estudio de los agentes causantes de lesiones en las edificaciones", desarrollada por el Instituto Tecnológico de Costa Rica y el Western Illinois University (WIU) de los Estados Unidos.

\section{Keywords}

Cultural heritage; traditional architecture; cultural property preservation; humidity; winds, bioclimatology.

\begin{abstract}
Fungi hinder the conservation processes of heritage assets since they cause their biodeterioration. The spores produced by these organisms, which can also generate health complications in the property users, depend on the humidity accumulated in the materials to germinate, therefore controlling environmental conditions is decisive if making the most of each intervention performed is wanted. Computer Fluid Dynamics (CFD) applications that have been traditionally used for energy simulations are being applied to determine air currents within spaces and thereby promote conservation strategies based on natural ventilation.

This article aims to show the methodology used for the analysis of air flows within the "Antigua Capitanía" of Port Limón in Costa Rica, using the AUTODESK FlowDesign software and the data analysis strategy developed by Víctor Fuentes Freixanet and Manuel Rodríguez Viqueira . This document has as framework the research project called "Conservation of the Caribbean Architecture in Costa Rica: use of advanced techniques to study biological agents responsible of damage in these buildings.", developed by Costa Rica Institute of Technology and Western Illinois University.
\end{abstract}




\section{Introducción}

Los hongos constituyen un problema para la conservación de bienes culturales, al causar el biodeterioro de los materiales con los que estos son construidos [1]. Las esporas producidas por los hongos son una de las principales causas de contaminación de los espacios de un edificio, ya que además de causar enfermedades y alergias en los usuarios, dañan los inmuebles y los objetos que estos contengan [2]. Las esporas viajan en el aire y se depositan en las superficies, sin embargo dependen del contenido de humedad en los materiales para poder germinar; es por esto que una adecuada ventilación puede ayudar a controlar la humedad relativa (HR), la temperatura ( $\mathrm{T}$ ) y el contenido de humedad presente en los distintos objetos o estructuras, reduciendo así la tasa de actividad microbiana [2].

El viento es el aire en movimiento como consecuencia, principalmente, de diferenciales de temperatura y presión [3]. La ventilación interna de un edificio es un sistema dinámico, en donde diversidad de organismos y partículas son transportados de un lugar a otro según las corrientes de aire existentes [4]. Estas corrientes pueden variar según la localización del inmueble, en cómo el viento ingresa y sale de los espacios, en los cambios de dirección que se presenten en el recorrido, la velocidad del viento, zonas de alta y baja presión, entre otros. [3]. Identificar y manipular estas corrientes de aire resultan de vital importancia, ya que además de brindar confort a los usuarios, respaldarían las intervenciones que se realicen a un inmueble de valor patrimonial.

Por las razones antes mencionadas, es que la dinámica de fluidos por computadora (CFD por sus siglas en inglés), que originalmente ha sido utilizada para simulaciones energéticas, se ha venido empleando para comprender el movimiento del aire alrededor de bienes históricos [5]. La CFD se refiere a los métodos necesarios para describir el comportamiento de fluidos en sistemas complejos mediante el uso de herramientas computacionales [5].

La ciudad de Limón, ubicada en la costa Caribe de Costa Rica, presenta importantes ejemplares de edificaciones construidas durante el periodo liberal costarricense (1870-1940) [6], las cuales se caracterizan por el uso intensivo de la madera, tanto en estructura primaria como en cerramientos [7]; así como por las modificaciones que se le hicieron a estilos arquitectónicos foráneos, como por ejemplo el victoriano, para obtener objetos arquitectónicos resistentes a las condiciones del clima local [8]. Dichos inmuebles hoy se encuentran en peligro debido a la alta tasa de demoliciones que sufren estas edificaciones patrimoniales, pero también al alto nivel de deterioro que evidencian las estructuras, muchos a causa de agentes biológicos.

Bajo este contexto surge el proyecto "Conservación de la arquitectura caribeña costarricense a partir de la aplicación de técnicas de avanzada para el estudio de los agentes causantes de lesiones en edificaciones", el cual busca resaltar las características distintivas de la arquitectura caribeña costarricense, así como identificar técnicas innovadoras que faciliten su conservación. El proyecto es financiado por la Vicerrectoría de Investigación y Extensión del Instituto Tecnológico de Costa Rica (TEC), y cuenta con la participación de profesionales y estudiantes de las escuelas de Arquitectura y Urbanismo e Ingeniería Forestal de la institución, así como de la Escuela de Ciencias Biológicas de la Western Illinois University (WIU) de los Estados Unidos.

Este documento muestra la metodología aplicada para determinar las corrientes de aire internas en los edificios identificados utilizando software de CFD, exponiendo como caso de estudio la Antigua Capitanía, inmueble de gran valor para la comunidad limonense que fue restaurado en el año 2012 y que hoy se utiliza como casa cultural de la ciudad.

\section{Materiales y métodos}

El primer paso consiste en consultar a las fuentes competentes la información relacionada a velocidad y dirección del viento en el sitio. En este caso, la información se obtiene del Instituto 
Meteorológico Nacional (IMN), el cual remite los datos registrados por la estación meteorológica ubicada en el Aeropuerto Internacional de Limón, ubicado a aproximadamente $3.3 \mathrm{~km}$ al sur del centro de la ciudad.

La velocidad del viento es un parámetro donde intervienen diversos factores que afectan su comportamiento [9], por lo que antes de ingresar la información al software de CFD, es importante realizar una serie de correcciones a los datos obtenidos por parte de la IMN para obtener resultados simulados más congruentes con el entorno del inmueble en estudio. Se emplea el método desarrollado por Víctor Fuentes Freixanet y Manuel Rodríguez Viqueira, en donde se aplican correcciones por efecto de rugosidad y altura a la velocidad del viento.

La cantidad de edificaciones y la altura de estas, el tipo de vegetación o incluso la falta de cobertura vegetal, son aspectos que constituyen la rugosidad del terreno, la cual modifica la velocidad del viento [9]. En terrenos muy rugosos, la capa límite (zona a partir de la cual el viento no es afectado por las particularidades del suelo) es muy alto, mientras que en zonas con muy poca rugosidad la capa límite es más baja [9]. Según la American Society of Heating, Refrigerating and Air-Conditioning Engineers (ASHRAE) [10], la disminución de velocidad por efecto de rugosidad se determina por la siguiente ecuación:

Donde:

$$
\text { Vref }=A_{0} * V m
$$

Vref $=$ Velocidad de referencia estimada $(\mathrm{m} / \mathrm{s})$

$A_{0}=$ Constante de rugosidad

Vmet $=$ Velocidad meteorológica media $(\mathrm{m} / \mathrm{s})$

La velocidad meteorológica es la media de la velocidad del viento facilitada por la estación meteorológica. La constante de rugosidad depende de la capa límite del sitio, y se puede aproximar mediante la siguiente ecuación:

Donde:

$$
A 0=1,291^{-0.005214(\delta-250))}
$$

$A_{0}=$ Constante de rugosidad

$\delta=$ Altura de capa límite $(\mathrm{m})$

Para simplificar la tarea de los cálculos a realizar, se utiliza la siguiente tabla basada en las recomendaciones de ASHRAE.

Una vez obtenida la velocidad con la corrección por rugosidad, se le debe aplicar además otra corrección por altura, según sea necesario. Usualmente, las estaciones meteorológicas se encuentran a 10m sobre el nivel del terreno, por lo que si los análisis se van a realizar a otra altura se debe emplear la siguiente ecuación:

$$
V_{H}=V_{m e t}\left[\frac{\delta_{m e t}}{H_{m e t}}\right]^{a m e t}\left[\frac{H}{\delta}\right]^{a} \quad[10]
$$

Donde:

$V_{H}=$ Velocidad de del viento a la altura $H(\mathrm{~m} / \mathrm{s})$

$\mathrm{V}_{\text {met }}=$ Velocidad meteorológica $(\mathrm{m} / \mathrm{s})$

$\delta_{\text {met }}=$ Capa de fricción en la estación $(m)$ 


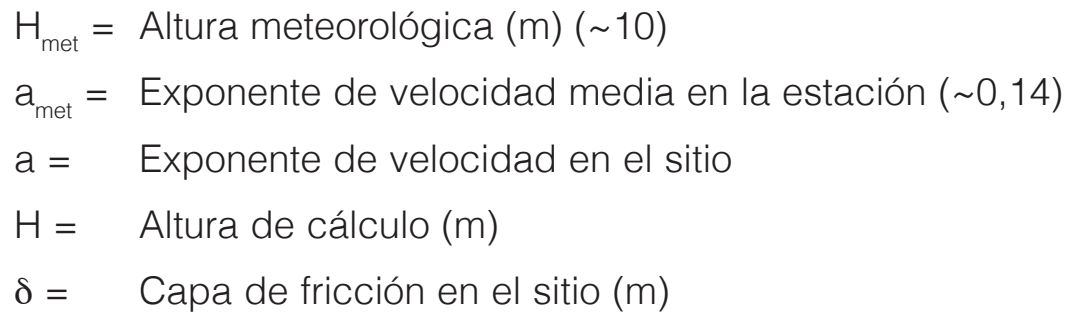

Cuadro 1. Constantes para calcular correcciones de velocidad de viento según capa límite.

\begin{tabular}{|c|c|c|c|c|c|}
\hline \multirow[t]{3}{*}{ Tipo de terreno } & $\begin{array}{c}\text { Alturas de } \\
\text { obstrucciones }\end{array}$ & $\begin{array}{l}\text { Capa de } \\
\text { fricción o } \\
\text { altura de } \\
\text { capa límite }\end{array}$ & $\begin{array}{c}\text { Constante } \\
\text { de } \\
\text { rugosidad }\end{array}$ & $\begin{array}{l}\text { Longitud de } \\
\text { rugosidad }\end{array}$ & $\begin{array}{c}\text { Exponente } \\
\text { de velocidad } \\
\text { media }\end{array}$ \\
\hline & & $\delta$ & & & $\alpha$ \\
\hline & (m) & (m) & & (m) & \\
\hline $\begin{array}{l}\text { Grandes centros urbanos en los que } \\
\text { al menos el } 50 \% \text { de los edificios tienen } \\
\text { una altura mayor de } 25 \mathrm{~m} \text { de, con una } \\
\text { distancia de al menos } 0.8 \mathrm{~km} \text { o } 10 \text { veces } \\
\text { la altura de la estructura de barlovento, } \\
\text { lo que sea mayor. }\end{array}$ & Mayor a 25 & 460 & 0,43 & 2 & 0,33 \\
\hline $\begin{array}{l}\text { Áreas urbanas o suburbanas, áreas } \\
\text { boscosas u otros terrenos con numerosos } \\
\text { espacios cercanos con obstrucciones } \\
\text { del tamaño de una vivienda unifamiliar } \\
\text { o más grande, en una distancia de } 460 \\
\text { m o } 10 \text { veces la altura de la estructura a } \\
\text { barlovento, lo que sea mayor. }\end{array}$ & $10-25$ & 370 & 0,7 & 0,65 & 0,22 \\
\hline $\begin{array}{l}\text { Terreno abierto con obstrucciones } \\
\text { dispersas con alturas generalmente } \\
\text { menores de } 10 \mathrm{~m} \text { incluyendo campo } \\
\text { abierto típico de los alrededores de una } \\
\text { estación meteorológica. }\end{array}$ & Menor a 10 & 270 & 1,16 & 0,05 & 0,14 \\
\hline $\begin{array}{l}\text { Zonas planas sin obstáculos expuestas } \\
\text { al viento, fluyendo sobre el agua por al } \\
\text { menos } 1.6 \mathrm{~km} \text {, o sobre una distancia } \\
\text { de } 460 \mathrm{~m} \text { o } 10 \text { veces la altura de la } \\
\text { obstrucción tierra adentro, lo que sea } \\
\text { mayor. }\end{array}$ & & 210 & 1,59 & 0,01 & 0,1 \\
\hline
\end{tabular}

Fuente [10]

Para la simulación de este proyecto se utilizó el software Flow Design, herramienta creada por AUTODESK para el análisis de fluidos en estudios arquitectónicos y mecánicos [11]. De cada planta se debe crear un modelo 3D en alguno de los formatos compatibles con el software, para poder ser importados. Flow Design determina el comportamiento de la volumetría al chocar con el aire, estableciendo así zonas de alta y baja presión, que configurarán los recorridos del aire al interior de los espacios. Es importante resaltar que en el modelo todas las aperturas deben ser huecas para permitir que el aire pase, ya que el software no reconoce superficies transparentes o permeables. 
Se debe modificar las unidades del software a metros $(\mathrm{m})$, e ingresar el dato de la velocidad del aire obtenido una vez aplicadas las correcciones por rugosidad y altura.

\section{Resultados}

El IMN reporta una velocidad de viento promedio para la ciudad de Limón de 2,2 m/s [12]. Este dato corresponde a las mediciones registradas por la entidad entre 2003 y 2014, las cuales fueron tomadas a una altura de $10 \mathrm{~m}$ con respecto al nivel del suelo en un aeropuerto. Es por esta razón que se emplea la metodología de Fuentes y Rodríguez para aplicar las correcciones de rugosidad y altura a la información suministrada.

Con respecto a la corrección por rugosidad, la velocidad meteorológica corresponde a 2,2m/s y la constante de rugosidad correspondiente al sitio, donde la mayoría de las construcciones son menores a 10m, es de 1,16; por lo que la velocidad de referencia es de 2,56 m/s.

Dado que la Antigua Capitanía es de dos plantas, se realizan ajustes de altura para cada nivel. Para el primer piso, considerando que la estructura se encuentra levantada al menos $0.5 \mathrm{~m}$ con respecto al nivel del suelo, se utiliza una altura de referencia de $2,5 \mathrm{~m}$, obteniendo como resultado una velocidad del viento de $1,73 \mathrm{~m} / \mathrm{s}$; mientras que para el segundo piso se utiliza una altura de referencia de $5,5 \mathrm{~m}$, dando como resultado un viento a 1,94m/s. Se debe considerar que el inmueble se encuentra levantado al menos $0,5 \mathrm{~m}$ del nivel de calle.

Como se aprecia en la figura 1, el edificio de la Antigua Capitanía está localizado en un lote esquinero y posee una distribución en "L", lo que le permite tener dos fachadas a vía pública (al este y sur), y un patio al noreste. El viento ingresa al inmueble desde el sureste, justo donde se ubica el parque y el mar, por lo que es un viento cargado de humedad.

La planta baja se compone de dos alas: la sur, que consiste en una planta libre donde actualmente hay cubículos de oficina y se realizan exposiciones; y la este, la cual se encuentra particionada en oficinas, bodegas y un espacio multiusos junto a la calle.

En cuanto al segundo nivel, cuenta con un balcón a lo largo de toda la fachada sur, justo frente al Parque Vargas. El espacio interno es más fragmentado que el del primer nivel, bridando a los usuarios diversos espacios de oficinas y alas independientes. Los baños se encuentran al centro de la planta, sin posibilidad de obtener ventilación o iluminación natural

El análisis en planta producido con AUTODESK Flow Design y mostrado en la figura 2, evidencia como, en el primer nivel, los recintos colindantes con las calles poseen un flujo de ventilación más elevado, mientras que los ubicados de forma adyacente al patio ven disminuido el flujo del aire; incluso el mismo patio presenta un detrimento considerable en la velocidad del viento con respecto a la galería frontal del inmueble. A nivel interno, también se observa la escasa ventilación en los recintos localizados al norte de la planta, así como en el sector oeste del ala sur de la Capitanía, espacio que hoy es utilizado como área de exhibición.

En ningún momento las corrientes de aire internas superan los $1.73 \mathrm{~m} / \mathrm{s}$, sino que, por el contrario, se van disminuyendo conforme entran a la propiedad. El gráfico también hace visible como el aire que sale al patio vuelve a ingresar al edificio por la puerta norte. La planta se rota en el software para que el flujo de aire de la simulación entre a la edificación por el mismo punto que lo hace en la realidad. 


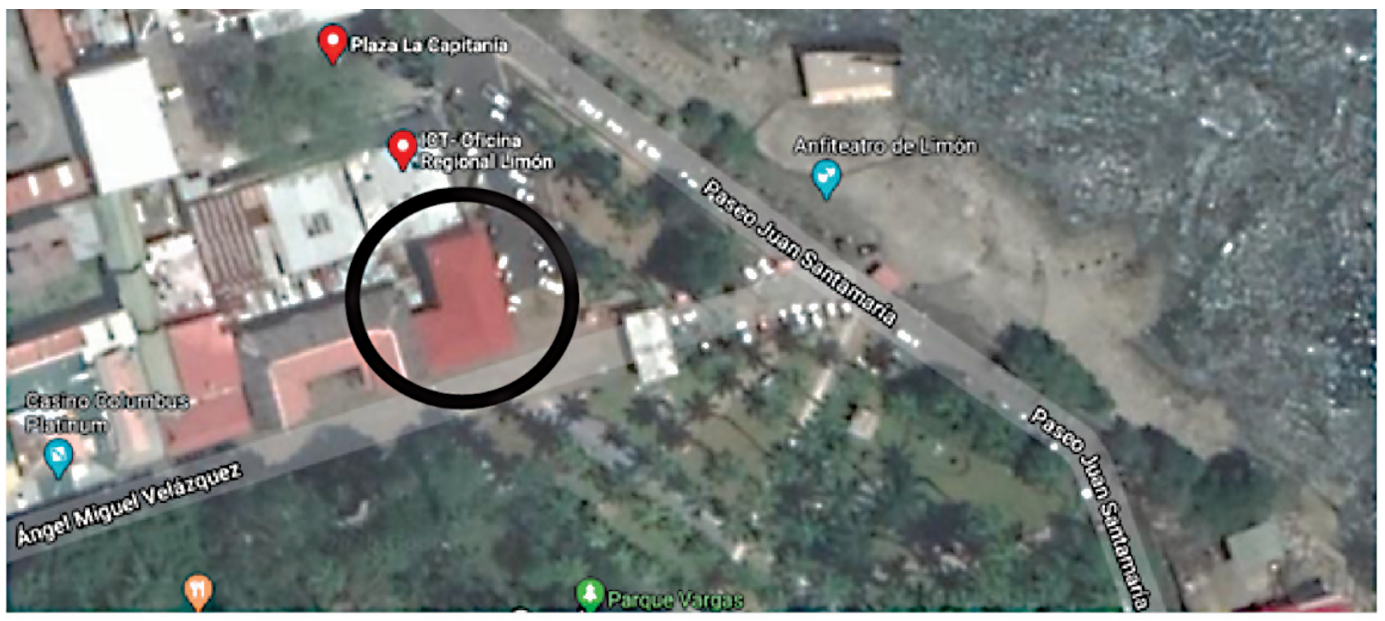

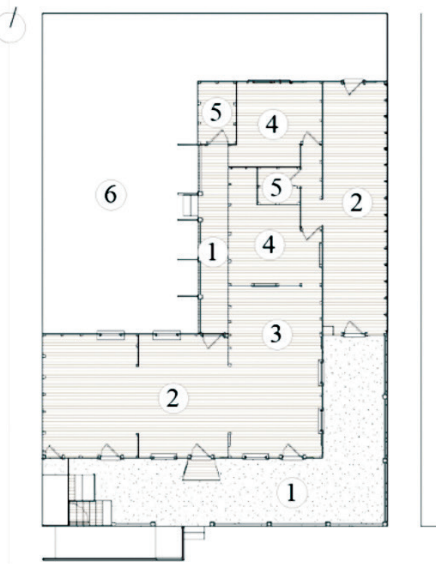

Primer nivel

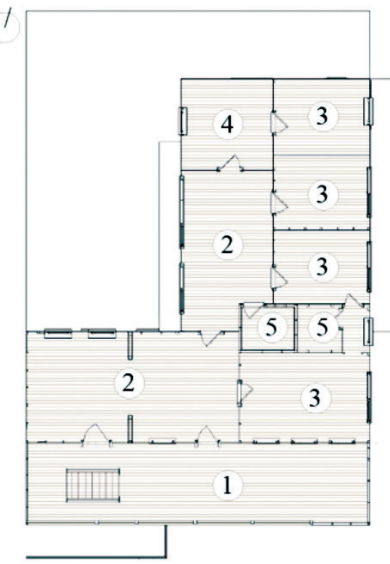

Segundo nivel
1- Galería / Balcón

2- Salones multiusos

3- Oficinas/Aulas

4- Bodegas

5- Baños

6- Patios

Figura 1. Ubicación de la Antigua Capitanía de Limón y plantas de distribución. Gráfico de elaboración propia con vista satelital tomada de Google Maps.

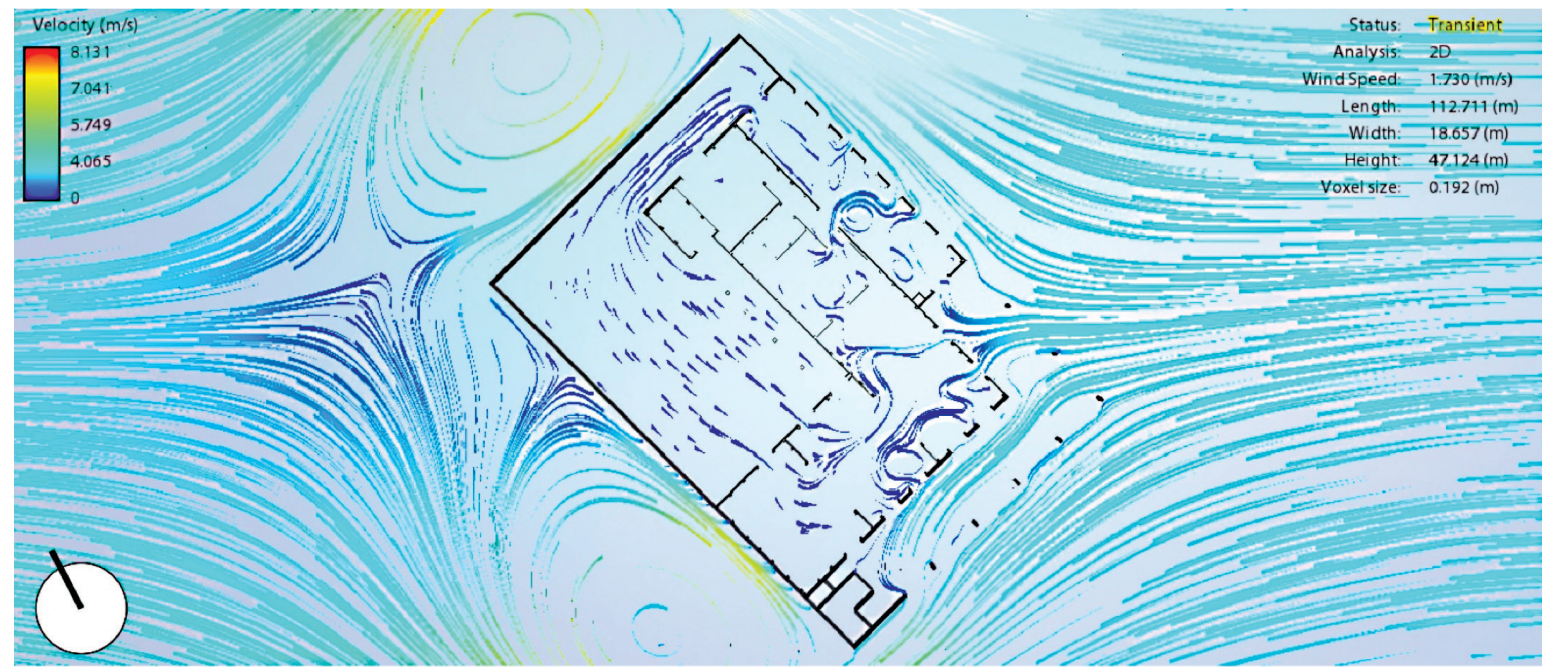

Figura 2. Flujos de aire en primer nivel de la Antigua Capitanía de Puerto Limón. Imagen de autoría propia elaborado con AUTODESK Flow Design 
En cuanto a los resultados del segundo nivel, la figura 3 muestra como los distintos recintos cuentan con corrientes de aire más rápidas, pero existen 3 espacios el centro de la planta que carecen de ventilación. El sector oeste del área multiusos presenta mayor flujo de viento con respecto a su homólogo del primer nivel. En esta planta, el aire que sale al patio no vuelve a ingresar a los espacios internos por ninguna de las aperturas al exterior.

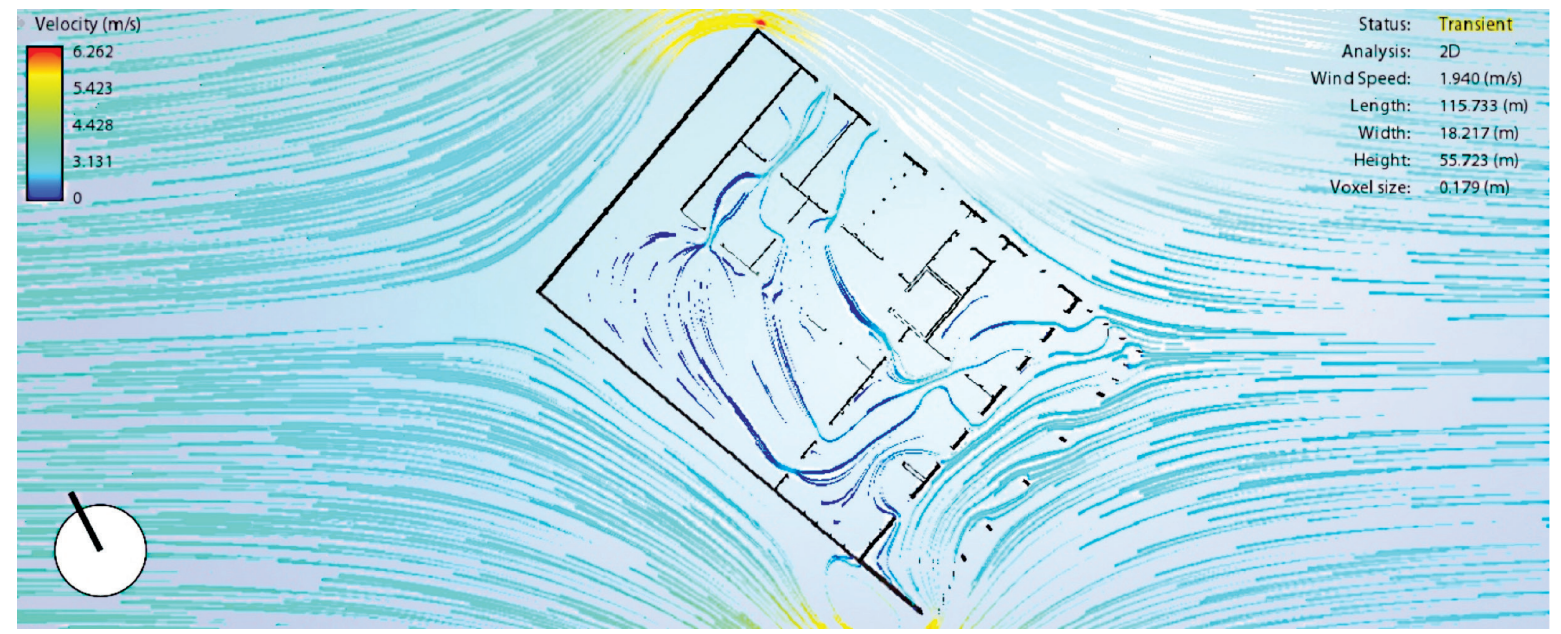

Figura 3. Flujos de aire en segundo nivel de la Antigua Capitanía de Puerto Limón. Imagen de autoría propia elaborado con AUTODESK Flow Design.

Con respecto al análisis de las envolventes, es importante tener claro que "el aire siempre se mueve de una presión alta a una presión baja" [3], por lo que la ubicación de las aperturas según las zonas de presión resulta un tema de estrategia. La figura 4 muestra como la interacción del viento con el edificio genera presiones oscilantes entre los 1.52 y $0.096 \mathrm{~Pa}$, siendo las columnas de madera y algunos cerramientos del balcón los que mayor presión presentan. Se empiezan a ver zonas de baja presión en la parte alta de las cubiertas. Las paredes donde se encuentran las ventanas no son las que presentan mayor presión principalmente las ubicadas al interior de la galería y balcón.

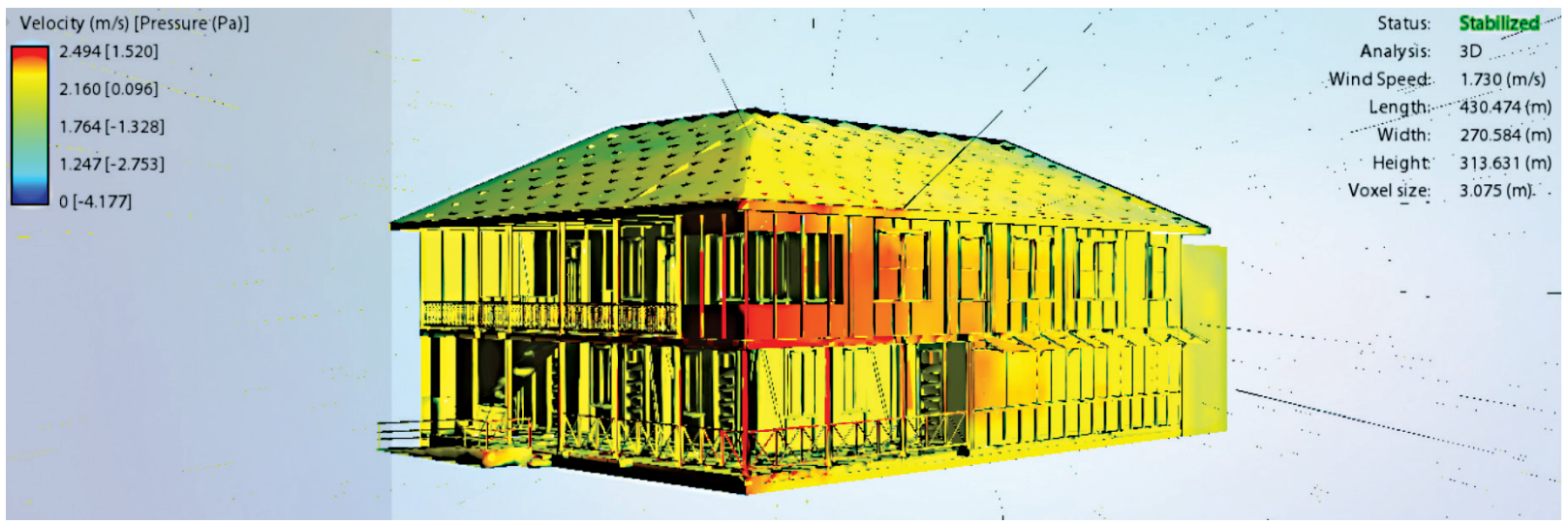

Figura 4. Zonas de presión en fachadas este y sur de la Antigua Capitanía de Puerto Limón. Gráfico generado con FlowDesign 
En la figura 5 se muestra que las zonas de presión en las fachadas que dan al patio de la propiedad oscilan entre los 0.097 y $-1.326 \mathrm{~Pa}$. Las partes de la cubierta ubicadas a sotavento son las que presentan menor presión, principalmente aquellas más cercanas a la cumbrera.

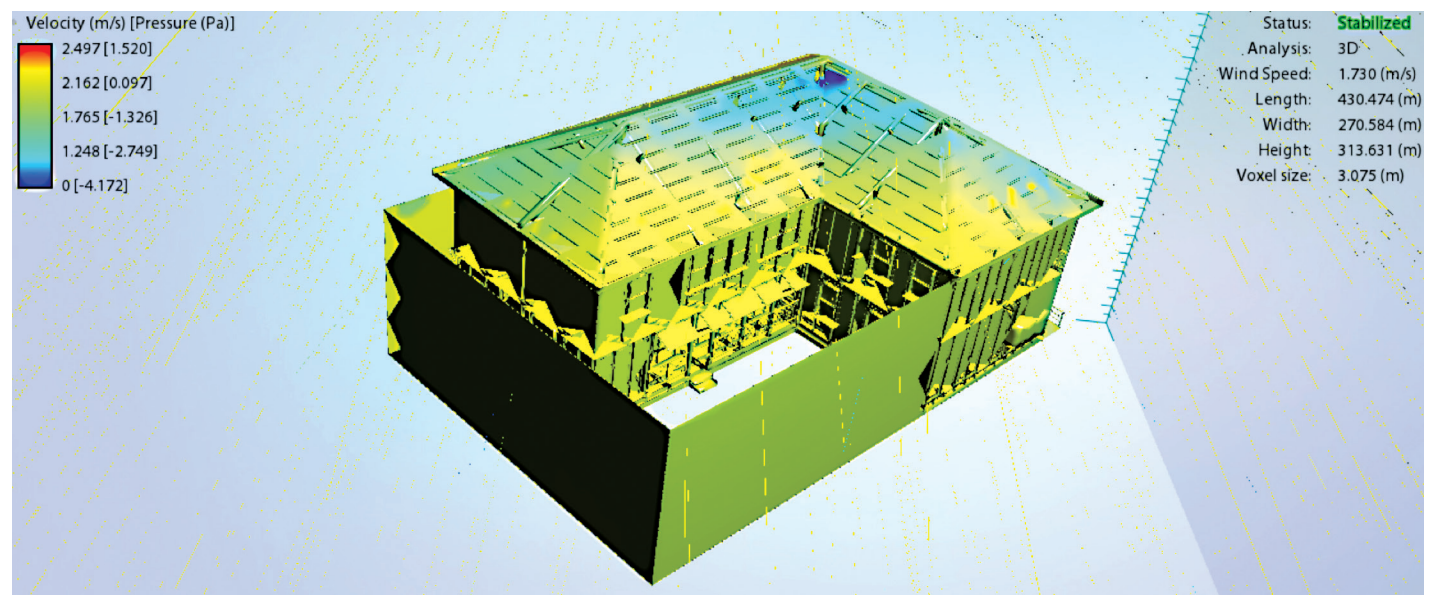

Figura 5. Zonas de presión en fachadas internas de la Antigua Capitanía de Puerto Limón. Gráfico generado con FlowDesign.

\section{Discusiones}

Los gráficos de las simulaciones en planta evidencian un menor flujo de aire en el nivel inferior, principalmente en los espacios ubicados al norte y oeste. En el caso del ala sur, no hay aperturas que permitan una adecuada ventilación hacia el costado oeste, lo que incide con la acumulación de humedad y generación de hongos verificada en sitio. Por el contrario, su espacio homólogo del segundo nivel presenta mejores condiciones de ventilación, esto debido a la ubicación de aperturas más cercanas a los bordes y a que el volumen de la escalera desaparece en este nivel. Los espacios con planta libre y aperturas en varios de sus extremos son los que mejor ventilación presentan. El patio interior, a pesar de estar al aire libre, se encuentra protegido por la estructura, por lo que la velocidad del aire es menor en comparación con la de la galería y el balcón. Su ubicación a barlovento debería generar una zona de succión que permita la ventilación cruzada.

A pesar de contar con una considerable cantidad de ventanas, las gráficas demuestran que los flujos de ventilación internos son cercanos a $0 \mathrm{~m} / \mathrm{s}$ en la mayoría de los espacios. Esta situación obedece a dos situaciones. La primera es la utilización de cerramientos para dividir los espacios, lo cual genera obstáculos al viento, haciéndole disminuir cada vez más su velocidad; y la segunda es que las presiones de las partes de las fachadas donde se encuentran las aperturas, tanto en las caras que reciben el viento como las del patio, presentan presiones muy similares, es decir, no hay un diferencial de presión que propicie la ventilación.

Las estancias ubicadas directamente en la fachada este, presentan flujos de ventilación medios o altos en comparación con los del resto del inmueble a pesar de ser áreas segregadas; esto se debe posiblemente a que sus ventanas están ubicadas en zonas con mayor presión.

Tanto las columnas como los muros bajos del balcón generan una especie de protección contra el viento, que impide que los cerramientos generen mayor presión y faciliten la entrada del aire.

Es importante notar que el análisis en los flujos de planta considera todas las puertas y ventanas abiertas, por lo que cerrar al menos una de ellas modificaría el resultado. 


\section{Conclusiones}

El análisis de flujos internos de ventilación por medio de CFD permite entender la relación del inmueble con su entorno, en este caso específico con el factor viento, para promover estrategias de conservación programada de los inmuebles. En el caso particular de la Antigua Capitanía del Puerto Limón, permite constatar que algunas zonas con alta presencia de humedad y agentes biológicos son también zonas donde los flujos de aire son bajos. Además, permite apreciar como algunas estrategias de división del espacio, necesarias para adecuar el inmueble a su nuevo uso, vienen en detrimento de la calidad del aire interno, situación que afecta tanto la conservación del inmueble como la calidad de vida de sus ocupantes.

El estudio desarrollado en este documento analiza el movimiento del aire a partir de la generación de diferenciales de presión, por lo que resultaría provechoso complementarlo con otro que profundice en los flujos de viento a partir de diferenciales de temperatura y la estratificación, si es que esta se diese en el espacio.

También, la investigación sienta los fundamentos para poder establecer estrategias de conservación que justifique futuras intervenciones, así como la generación de manuales de buenas prácticas en cuanto al uso dispositivos de climatización pasiva por parte de sus ocupantes.

\section{Referencias}

[1] K. Sterflinger, «Fungi: Their role in deterioration of cultural heritage,» Fungal Biology Reviews, vol. 24, pp. 47-55, 2010.

[2] N. Valentín, «Microbial contamination in archives and Museums: Health hazards and preventive strategies using air ventilation systems,» 2007.

[3] V. Fuentes Freixanet, «Arquitectura Bioclimática, ventilación natural.,» 2015.

[4] E. Di Carlo, R. Chisesi, B. Giovanna, S. Barbaro, G. Lombardo, R. Valentina, M. Sebastianelli, T. Giovanni y F. Palla, «Fungi and Bacteria in Indoor Cultural Heritage Environments: Microbial-r elated Ri sks for Artworks and Human Health,» Environment and Ecology Research, vol. 4, n 5, pp. 257-264, 2016.

[5] J. Grau-Bové, L. Mazzei, M. Strlic y M. Cassar, «Fluid simulations in heritage science,» Heritage Science, vol. 7, $\mathrm{n}^{\circ} 16,2019$.

[6] I. Hernández Salazar, D. Porras Alfaro y K. García Baltodano, «La arquitectura caribeña costarricense en la trama urbana histórica de la ciudad de Limón entre el periodo de 1871-1920,» de Tercer Congreso Internacional Hispanoamericano de Historia de la Construcción, Ciudad de México, 2019.

[7] O. Sanou y F. Quesada, «Orden, Progreso y Civilización, 1871-1914. Transformaciones urbanas y arquitectónicas,» de Historia de la Arquitectura de Costa Rica, E. Fonseca y J. E. Garbier, Edits., San José, Museos del Banco Central y Centro de Investigaciones Históricas de Centroamérica, 1998, pp. 219-317.

[8] R. Woodbridge, Historia de la arquitectura en Costa Rica, Cartago: Editorial Tecnológica de Costa Rica, 2003.

[9] V. A. Fuentes Freixanet y M. Rodríguez Vaqueira, Ventilación natural: cálculos básicos para arquitectos, Ciudad de México: Universidad Autónoma Metropolitana, 2004.

[10] American Society of Heating, Refrigerating and Air-Conditioning Engineers, "ASHRAE Handbook, Fundamentals,» ASHRAE, Atlanta, 2017.

[11] A. Baghaei Daemei, E. Mehrinejad Khotbehsara, E. Malekian Nobarani y P. Bahrami, «Study on wind aerodynamic and flow characteristics of triangular-shaped tall buildings and CFD simulation in order to assess drag coefficient,» Ain Shams Engineering Journal, vol. 10, n³ 3, pp. 541-548, 2019.

[12] Instituto Meteorológico Nacional, «PROMEDIOS MENSUALES DE DATOS CLIMATICOS,» 2019. 